\title{
Taking into account the non-proportional loading effect on high cycle fatigue life predictions obtained by invariant-based approaches
}

\author{
Lorenzo Bercelli ${ }^{1}$, Cédric Doudard ${ }^{1}$, and Sylvain Moyne ${ }^{1}$ \\ ${ }^{1}$ ENSTA Bretagne, UMR CNRS 6027, IRDL, F-29200, Brest, France
}

\begin{abstract}
Industrial structures are often subjected to multiaxial fatigue loadings. If the multiple stress signals are not synced the loading is said to be non-proportional. Most of the multiaxial fatigue criteria give highly inaccurate lifetime predictions when used in the case of such loadings. The scalar equivalent stress defined by the criteria does not take into account the non-proportional nature of the multiaxial loading and leads to nonconservative predictions. Moreover a multiaxial fatigue criterion can only be applied on a stress cycle which has no clear definition when multiple unsynced signals are to be considered. This study addresses these issues by proposing a correction of an invariant based multiaxial fatigue criterion through the definition of a non-proportional degree indicator. A definition of multiaxial cycle is also given based on the Wang-Brown method. Finally a complete chain of invariant based lifetime prediction for nonproportional multiaxial fatigue is validated.
\end{abstract}

\section{Introduction}

A large number of multiaxial fatigue criteria are proposed in literature to determine the high cycle fatigue lifetime of a structure. Most of these criteria are calculated on specific stress cycles extracted from the loading signal using a cycle breakdown algorithm. In the case of proportional multiaxial signal, meaning it can be represented by a time dependent scalar, cycles can be extracted using the Rainflow counting [1]. This method cannot be used in the case of a Non-Proportional (NP) signal for which no scalar variable is representative of the stress state. The Wang Brown counting [2] is an invariant based algorithm that extracts multiaxial half-cycles from the multiaxial signal. Nevertheless once a NP cycle is defined, most multiaxial fatigue criteria do not give accurate predictions. Citeria such as Sines [3] make use of the second invariant of the alternate deviatoric stress tensor $\mathrm{J}_{2 \mathrm{a}}$ which can be defined as the radius of the Minimum Enclosing Ball (MEB) 
containing the stress signal represented in the deviatoric space. This definition does not necessarily take into account the multiaxial nature of the signal. A counter example is the case of a 2D proportional loading with a length $\mathrm{L}$ in the deviatoric space, being identical in the sense of the MEB method to a circular NP loading with a diameter of L. From this observation a few multiaxial models try to take into account the NP nature of the loading. A detailed overview is available in the work of Peikowski [4]. A few of those models are based on a critical plane approach, as in the work of Kanazawa et al. [5] in which the shift angle between normal and shear strains is considered. Fatemi and Socie [6] assumed that the normal stress of the critical plane already takes into account the effects of nonproportionality. In Li et al. [7] NP parameter is based on a critical plane approach in which the projected shear stress amplitude is integrated in a discretized space. A few other models differ from the critical plane approach, as in Anes et al. [8] where a material constant considers the fatigue performance drop between proportional and NP loadings. An invariant based approach such as the proposed by $\mathrm{Vu}$ et al. [9] relies on the use of the mean value of $\mathrm{J} 2 \mathrm{a}$ on one loading cycle as indicator.. The goal of the present study is to provide a NP indicator that is material independent, invariant based and adapted to high cycle fatigue. The integration of this indicator in a complete procedure of lifetime prediction is also demonstrated. Firstly, the effect of NP degree on the accuracy of the Sines [3] multiaxial fatigue criterion is presented. Then a NP indicator is defined to correct the criterion on an experimental database. Finally the corrected criterion is used to predict fatigue lifetimes and the results are compared to experimental results.

\section{Multiaxial fatigue criteria in the NP case}

When NP loadings are considered, most multiaxial fatigue criteria give erroneous results $[4 ; 10]$. This effect is demonstrated here through Sines criterion [3] using experimental fatigue data collected by Weber [11].

\subsection{Multiaxial fatigue criteria}

The Sines criterion can be defined by an equivalent scalar stress $\sigma_{e q}$ that is compared to a material constant acting as a failure threshold. It is defined as follow

$$
\sigma_{e q}=J_{2 a}+\alpha_{S} I_{1 m} \leq \beta_{S}
$$

where $J_{2 a}$ is the amplitude of the second invariant of the deviatoric stress tensor (defined as the radius of the MEB of the cycle in the deviatoric space), $I_{1 m}$ is the mean value of the stress tensor first invariant. The parameters $\alpha_{S}$ and $\beta_{S}$ can be identified when using the criterion on simple $1 \mathrm{D}$ loadings such as repeated tension loading $(\mathrm{R}=0)$ and fully reversed torsion loading $(\mathrm{R}=-1)$. The parameters are determined as follow

$$
\left\{\begin{array}{c}
\alpha_{S}=2 \sqrt{3} \frac{\tau_{-1}}{\sigma_{0}}-1 \\
\beta_{S}=\sqrt{3} \tau_{-1} \text { or } \beta_{S}=\sigma_{-1}
\end{array}\right.
$$

where $\sigma_{0}$ is the fatigue limit in repeated tension, $\tau_{-1}$ is the fatigue limit in fully reversed torsion and $\sigma_{-1}$ is the fatigue limit in fully reversed tension 


\subsection{Experimental database}

Each criterion proposed in literature is more or less adapted to a given loading type (high mean stress, bi-axial tension, ...). In this study only fully reversed loadings (no mean stress) are considered. Experimental results collected by Weber [11] are used : fatigue limit in tension-torsion fatigue tests are given for many different metallic materials. The stress tensor $\underline{\underline{\sigma}}(t)$ in fully reversed tension torsion can be expressed as follow

$$
\underline{\underline{\sigma}}(t)=\left[\begin{array}{ccc}
\sigma_{a} \sin (\omega t) & \tau_{a} \sin (\omega t+\varphi) & 0 \\
\tau_{a} \sin (\omega t+\varphi) & 0 & 0 \\
0 & 0 & 0
\end{array}\right]
$$

with $\sigma_{a}$ the normal stress amplitude and $\tau_{a}$ the shear stress amplitude. A $0^{\circ}$ phase difference $\varphi$ stands for a proportional loading meaning the stress tensor can be represented by a time dependent scalar $\mathrm{f}(\mathrm{t})$, while a non-zero phase difference implies a nonproportionality in which case no scalar function $\mathrm{f}(\mathrm{t})$ exists. This can be expressed as follows

with

$$
\left\{\begin{array}{l}
\text { if } \varphi=0^{\circ} \rightarrow \underline{\underline{\sigma}}(t)=\mathrm{f}(\mathrm{t}){\underline{\underline{\sigma_{c}}}} \\
\text { if } \varphi \neq 0^{\circ} \rightarrow \underline{\underline{\sigma}}(t) \neq \mathrm{f}(\mathrm{t}) \underline{\underline{\underline{\sigma_{c}}}}
\end{array}\right.
$$

$$
\underline{\underline{\sigma_{c}}}=\left[\begin{array}{ccc}
\sigma_{a} & \tau_{a} & 0 \\
\tau_{a} & 0 & 0 \\
0 & 0 & 0
\end{array}\right]
$$

Thus, the phase difference $\varphi$ is an NP indicator. The case of a circular signal in the 2D deviatoric space gives the highest NP degree possible, obtained when $\varphi=90^{\circ}$ and $\sigma_{a}=\sqrt{3} \tau_{a}$

\subsection{Criteria error for a NP loading}

A multiaxial fatigue criterion can be applied on any multiaxial signal (or cycle) but a significant error can be observed for NP loadings [8], [12]. The error of a criterion $e_{r r}$ can be defined as

$$
e_{r r}=\frac{\beta-\sigma_{e q}}{\beta}
$$

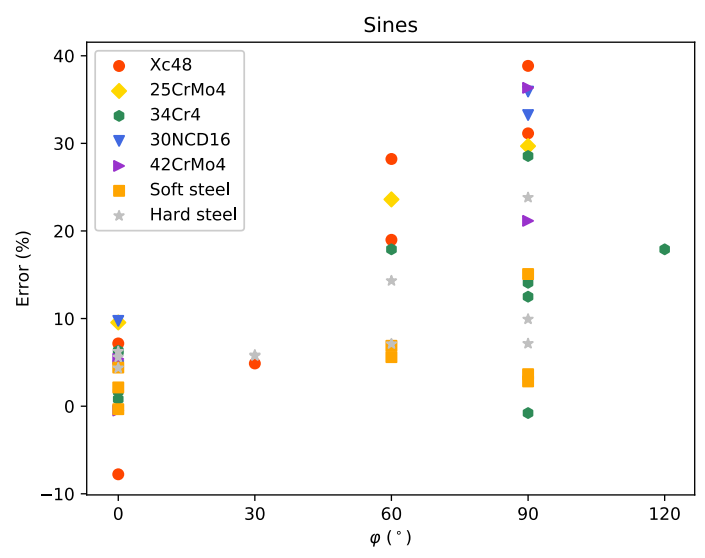

Fig. 1. Sines criterion error for NP loadings, corresponding material in legend 
A negative error would lead to a conservative prediction, while a positive error leads to non-conservative predictions. Based on Weber's experimental database, the error of the Sines criterion can be plotted as a function of the phase difference. Fig. 1 shows that the closer the phase is to $90^{\circ}$, the greater the error: the Sines criterion accuracy is dependent of the NP degree. Nevertheless the phase difference is not a good NP degree indicator as the ratio $\sigma_{a} / \tau_{a}$ also has an influence. In order to be able to correct the criteria in the case of a NP loading, an accurate indicator of the NP degree is required.

\section{Criteria correction following a NP indicator}

The shift angle between stress signals does not accurately represent the NP degree of a multiaxial signal as the stress amplitude ratio is also to be considered. Moreover a real random multiaxial signal can hardly be defined by a simple shift angle. In this part a new NP indicator, $\mathrm{I}_{\mathrm{NP}}$, is defined and incorporated in the criterion. $\mathrm{I}_{\mathrm{NP}}$ is constructed to respect the following constraints:

- be material independent

- be compatible with invariant based multiaxial criteria

- be adapted for high cycle fatigue regime

- $\quad$ be defined for any multiaxial signal

- $\quad$ have 0 and 1 has lower bound and upper bound respectively

- be independent of the running direction in time of the signal.

\subsection{Definition of a NP indicator}

The NP indicator presented in this paper is based on geometric considerations of the multiaxial signal in the deviatoric space with the Von Mises norm, $\mathrm{J}_{2}$. In the tension-torsion case, a proportional multiaxial signal constitutes a straight line, for which the indicator value should be 0 . The most NP case corresponds to a circle in the deviatoric space, for which the indicator value should be 1 (Fig. 2).
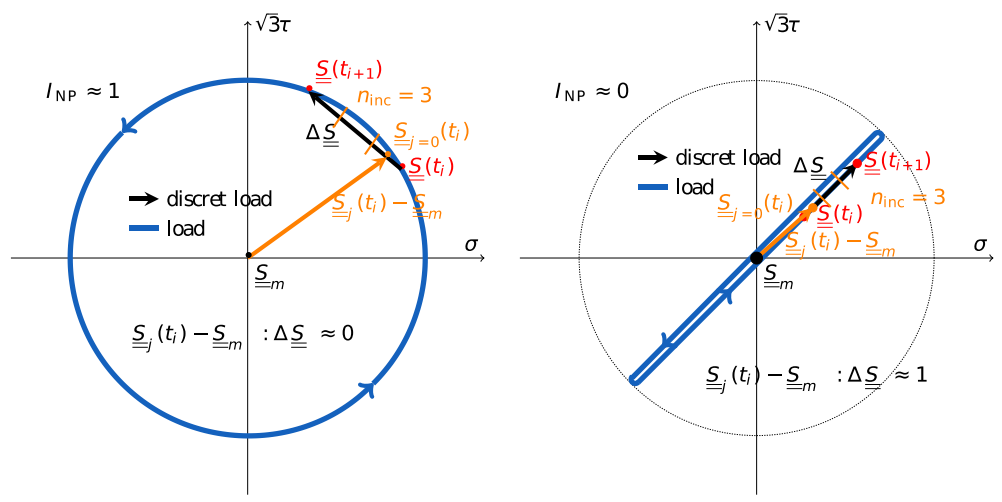

Fig. 2. NP an proportional loadings in a $2 \mathrm{D}$ deviatoric space 
The expression of the indicator for a loading cycle between $t_{0}$ and $t_{n}$ is given by

$$
I_{N P}=1-\frac{1}{L_{s}} \int_{t_{0}}^{t_{n}} \frac{\left|\left(\underline{\underline{s}}(t)-\underline{\underline{s}}_{m}\right): \frac{d \underline{s}}{d t}\right|}{\sqrt{\left(\underline{\underline{s}}(t)-\underline{\underline{s}}_{m}\right):\left(\underline{\underline{s}}(t)-\underline{\underline{s}}_{m}\right)}} d t
$$

with $L_{s}$ the length of the signal, $\underline{\underline{s}}$ the deviatoric stress tensor and $\underline{\underline{s}}_{m}$ the tensor of the mean deviatoric stress in each direction. In practice the stress signal is discrete and the following definition of $\mathrm{I}_{\mathrm{NP}}$ is preferred

$$
I_{N P}=1-\frac{1}{L_{s}} \sum_{t_{i}}^{\left[t_{0}, \ldots, t_{n-1}\right]} \frac{1}{n_{\text {inc }}} \sum_{j=0}^{n_{\text {inc }}-1} \frac{\left|\left(\underline{\underline{s}}_{j}\left(t_{i}\right)-\underline{\underline{s}}_{m}\right): \Delta \underline{\underline{s}}\right|}{\sqrt{\left(\underline{s}_{j}\left(t_{i}\right)-\underline{\underline{s}}_{m}\right):\left(\underline{\underline{s}}_{j}\left(t_{i}\right)-\underline{\underline{s}}_{m}\right)}}
$$

with $\underline{\underline{s}}_{j}\left(t_{i}\right)=\frac{1}{2 n_{\text {inc }}}\left((2 j+1) \underline{\underline{s}}\left(t_{i}\right)+\left(2 n_{\text {inc }}-2 j-1\right) \underline{\underline{s}}\left(t_{i}\right)\right)$ the barycentric mean of the deviatoric stress at $\mathrm{j}$ between $\mathrm{t}_{\mathrm{i}}$ and $\mathrm{t}_{\mathrm{i}+1}$.

\subsection{Criterion correction}

The previous indicator allows the estimation of an invariant based NP degree. The error of Sines is plotted against $\mathrm{I}_{\mathrm{NP}}$ and a correction is proposed, based on the data from $\S 2.3$. The error grows with the value of the indicator $I_{N P}($

Fig. 3) meaning the more the signal is NP the more inaccurate is the prediction. An important dispersion exists because the data mixes many different materials from different publications. A linear model has been chosen to estimate the error,

$$
e_{r r}\left(I_{N P}\right)=a_{c o r r} I_{N P}
$$

The criterion is considered to give a null error for proportional loadings.

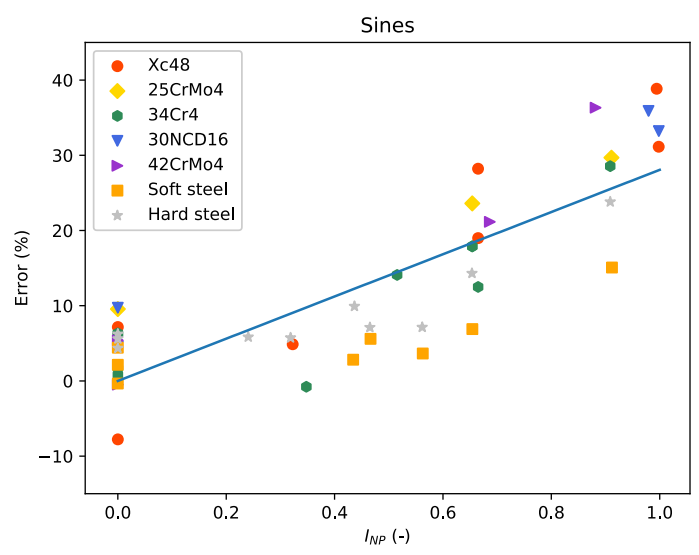

Fig. 3. Sines error according to $I_{N P}$

When corrected the criterion gives errors between $12 \%$ and $-12 \%$, most being negative which is to say conservative. The correction parameter is identified : $a_{\text {corr }}=0.28$. The original definition of the criterion as given in equation 1 is now distinguished from the corrected definition

$$
\sigma_{e q} \leq \beta\left(1-a_{c o r r} I_{N P}\right)
$$




\section{Validation of the corrections on an independent database}

Using the corrected definition of Sines criterion (equation 10), life predictions in high cycle fatigue are evaluated and compared to experimental results from another experimental database provided by Anes et al. [8;13]. A multiaxial cycle counting method is used based on the Wang-Brown method as presented by Meggiolaro et al. [12].

\subsection{Validation database}

The experimental database used for validation of the corrected criteria is based on results presented by Anes et al. throughout their work in different publications $[8 ; 13 ; 14]$. NP tension-torsion fatigue tests on $42 \mathrm{CrMo} 4$ samples under a wide range of amplitudes are performed in their work. The number of different loading types as well as the complete information on the $42 \mathrm{CrMo} 4$ material are the reasons for the choice of Anes et al. database. Seven loading types are explored (Fig. 4). It is worth to notice that only Case1 to Case 3 are unicyclic signals in the sense of the Wang-Brown counting method.
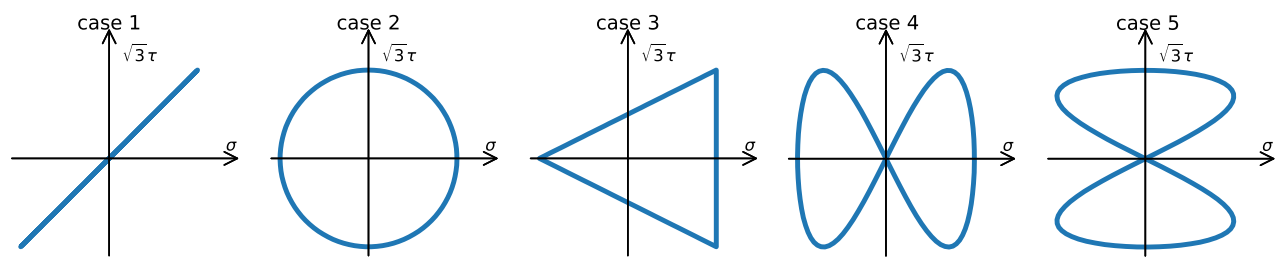

Fig. 4. Validation loadings from V. Anes et al. database [8;13]

\subsection{Multiaxial cycle breakdown}

Since Case 4 to Case 5 are not unicyclic, it is necessary to extract cycles using the Wang Brown method as modified by Meggiolaro et al. [12]. This method considers the multiaxial signal in the deviatoric space with $\mathrm{J}_{2}$ as norm through a geometric algorithm. This allows the definition of multiple half-cycles that can eventually be combined in full cycles. Some remaining half-cycles are to be considered (in the same way the Rainflow counting leaves a residue). When applied to a proportional signal the Wang-Brown method gives the exact same results as the Rainflow counting. This gives good confidence in the NP cycles breakdown. Moreover the Wang-Brown method uses the $J_{2}$ norm and in this sense is coherent with both Sines criterion and $\mathrm{I}_{\mathrm{NP}}$.

\subsection{Finite life prediction}

Multiaxial fatigue criterion can be used to predict the number of cycles to failure for each specific loading. This is achieved by writing

$$
\left\{\begin{array}{c}
\beta_{S}(N)=A_{S} N^{B} \\
\alpha_{S}(N)=\alpha_{S}
\end{array}\right.
$$

with $A_{S}=A_{\tau_{-1}} \frac{\sigma_{-1}}{\tau_{-1}}, A_{\tau_{-1}}$ and $B$ being the Basquin parameters of the material. Then, for a given cycle, the lifetime is deduced from equations 10 and 11 
and for a half-cycle :

$$
N_{c}=\left(\frac{\sigma_{e q_{i}}}{\left(1-a_{c o r r} I_{N P}\right) A_{S}}\right)^{1 / B}
$$

$$
N_{h}=\frac{1}{2}\left(\frac{\sigma_{e q_{i}}}{\left(1-a_{c o r r} I_{N P}\right) A_{S}}\right)^{1 / B}
$$

The Miner rule is used to determine $\mathrm{N}_{\text {tot }}$ as the number of full signal repetition before failure

$$
N_{\text {tot }}=1 /\left(\sum_{\text {half cycles }} \frac{1}{N_{h}}+\sum_{\text {cycles }} \frac{1}{N_{c}}\right)
$$

It is worth to notice that each cycle or half cycle for Case4 to Case 5 is corrected independently, meaning it is not corrected according the value of $\mathrm{I}_{\mathrm{NP}}$ of the whole signal but of each corresponding cycle or half cycle. The parameters of the Basquin law as well as the material parameters are extracted from the work of Anes et al. on the 42CrMo4 and Weber [11] (Table 1).

Table 1 Material data of the $42 \mathrm{CrMo} 4$

\begin{tabular}{l|l|l|l|l|l} 
Parameter & $\boldsymbol{\sigma}_{-1}(\mathbf{M P a})$ & $\boldsymbol{\tau}_{-\mathbf{1}}(\mathbf{M P a})$ & $\boldsymbol{\sigma}_{\mathbf{0}}(\mathbf{M P a})$ & $\mathbf{A}_{\tau-1}(\mathbf{M P a})$ & $\mathbf{B}_{\mathbf{S}}=\mathbf{B}_{\mathbf{C}}$ \\
\hline Value & 398 & 260 & 620 & 864.78 & -0.061 \\
\hline Reference & {$[14]$} & {$[14]$} & {$[11]$} & {$[14]$} & {$[14]$}
\end{tabular}

\subsection{Results}

For each loading test the number of cycles to failure is calculated according to equation 12. Preliminary to that a Wang-Brown cycle breakdown is applied for Case 4 to Case5. The predicted number of cycles is then compared to the experimental results (Fig. 5). The corrected Sines criterion show significantly improved results compared to the noncorrected version.

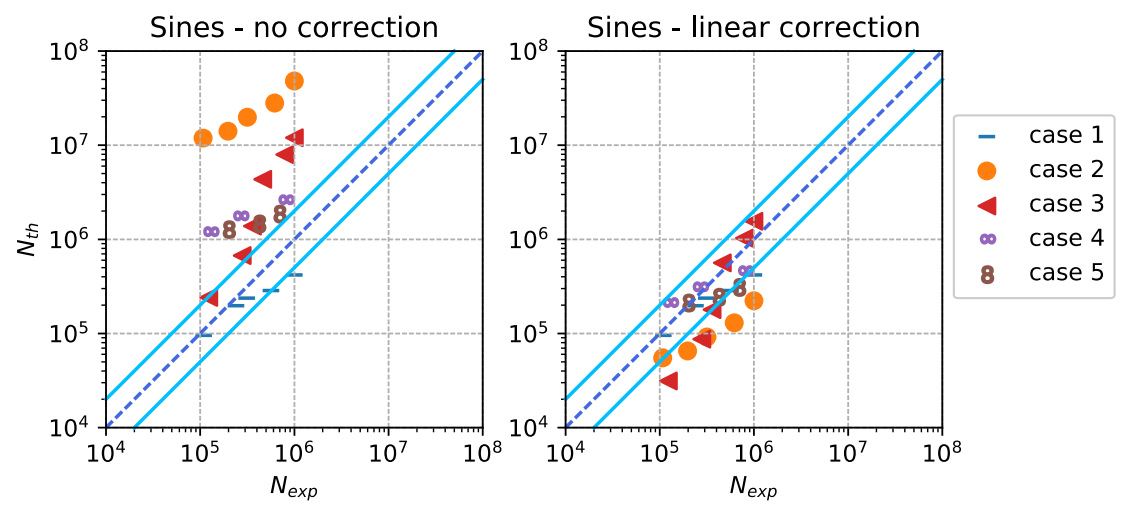

Fig. 5. Results of a linear correction with $\mathrm{I}_{\mathrm{NP}}$ on Sines criterion

In Table 2 is detailed the values of $\mathrm{I}_{\mathrm{NP}}$ of each half-cycle and cycle for Case 4 to Case5. 'Full Signal' gives the NP degree for the full signal as if it was considered to be one unique cycle. 'Assembly' gives a total NP degree according to the following sum

$$
I_{N P_{\text {Assembly }}}=\sum_{i} \frac{l_{i} I_{N P_{i}}}{\sum_{j} l_{j}}
$$


with $I_{N P_{i}}$ the indicator value for one cycle or half-cycle and $l_{i}$ the length of a cycle/halfcycle according to the $\mathrm{J}_{2}$ norm. It can be noted that $I_{N P_{\text {signal }}} \neq I_{N P_{\text {assembly }}}$ meaning the Wang-Brown breakdown affects the NP degree of a signal. This is because the mean value $\underline{\underline{S}}_{m}$ is used to calculate $\mathrm{I}_{\mathrm{NP}}$ and this value is not the same for a half-cycle and for the full signal.

Table 2 Mean value of $\mathrm{I}_{\mathrm{NP}}$ for each loading case

\begin{tabular}{lll|lll}
\hline $\begin{array}{l}\text { Loading } \\
\text { case }\end{array}$ & $\begin{array}{l}\text { Cycle } \\
\text { breakdown }\end{array}$ & $\begin{array}{l}\mathbf{I}_{\mathbf{N P}} \\
\text { Sines }\end{array}$ & $\begin{array}{l}\text { Loading } \\
\text { case }\end{array}$ & $\begin{array}{l}\text { Cycle } \\
\text { breakdown }\end{array}$ & $\begin{array}{l}\mathbf{I}_{\mathbf{N P}} \\
\text { Sines }\end{array}$ \\
\hline Case5 & Full Signal & 0.364 & Case6 & Full Signal & 0.364 \\
& Cycle 1 & 0.422 & & Cycle 1 & 0.422 \\
& Half-Cycle 1 & 0.081 & & Half-Cycle 1 & 0.081 \\
& Half-Cycle 2 & 0.165 & & Half-Cycle 2 & 0.165 \\
& Assembly & 0.326 & & Assembly & 0.324 \\
\hline
\end{tabular}

\section{Conclusions}

In this study a new invariant based NP indicator is defined and integrated in a full high cycle fatigue lifetime prediction chain based on Sines criterion. Using a complete experimental database this indicator can be used to propose a corrected Sines criterion. Based on another experimental database for multiple NP signals and using the WangBrown cycle breakdown method the accuracy of the corrected criterion is demonstrated. The results (Fig. 5) show considerable improvements over the original uncorrected approach, both for simple unicyclic signals as well as more complex signals. It should be noted that the Wang-Brown method alters the NP degree of a multiaxial signal as the weighted average of $I_{N P}$ for each cycle is not equal to the value of $I_{N P}$ for the global signal. To achieve an even better correction of the criterion a more sound experimental database could be established. Indeed, as the data collected by Weber [11] are obtained from tests performed between 1945 and 1992, we can assume that the test protocols vary significantly.

\section{References}

1. Fatigue sous sollicitations d'amplitude variable. Méthode Rainflow de comptage des cycles. Principe et utilisation. (AFNOR, 1993).

2. Wang, C. H. \& Brown, M. W. On plastic deformation and fatigue under multiaxial loading. Nucl. Eng. Des. 162, 75-84 (1996).

3. Sines, G. \& Ohgi, G. Fatigue Criteria Under Combined Stresses or Strains. J. Eng. Mater. Technol. 103, 82 (1981).

4. Pejkowski, Ł. On the material's sensitivity to non-proportionality of fatigue loading. Archives of Civil and Mechanical Engineering 17, 711-727 (2017).

5. K. Kanazawa, K. J. Miller, M. W. B. Cyclic Deformation of $1 \% \mathrm{Cr}-\mathrm{Mo}-\mathrm{V}$ Steel under Out-of-Phase Loads. Fatigue Fract. Eng. Mater. Struct. 2, 217-228 (2017).

6. Fatemi, A. \& F. Socie, D. A critical plane approach to multiaxial fatigue damage including out-phase loading. Fatigue Fract. Engng. Mater. Struct. 11, 149-165. Fatigue Fract. Eng. Mater. Struct. 11, 149-165 (1988).

7. Li, B. C., Jiang, C., Han, X. \& Li, Y. A new approach of fatigue life prediction for 
metallic materials under multiaxial loading. Int. J. Fatigue 78, 1-10 (2015).

8. Anes, V., Reis, L., Li, B. \& De Freitas, M. New approach to evaluate nonproportionality in multiaxial loading conditions. Fatigue Fract. Eng. Mater. Struct. 37, 1338-1354 (2014).

9. Vu, Q. H., Halm, D. \& Nadot, Y. Multiaxial fatigue criterion for complex loading based on stress invariants. Int. J. Fatigue 32, 1004-1014 (2010).

10. Meggiolaro, M. A. \& De Castro, J. T. P. An improved multiaxial rainflow algorithm for non-proportional stress or strain histories - Part I: Enclosing surface methods. Int. J. Fatigue 42, 217-226 (2012).

11. Weber, B. Fatigue multiaxiale des structures industrielles sous chargement quelconque. 243 (1999).

12. Meggiolaro, M. A. \& de Castro, J. T. P. An improved multiaxial rainflow algorithm for non-proportional stress or strain histories - Part II: The Modified Wang-Brown method. Int. J. Fatigue 42, 194-206 (2012).

13. Anes, V., Reis, L., Li, B. \& De Freitas, M. New cycle counting method for multiaxial fatigue. Int. J. Fatigue 67, 78-94 (2014).

14. Anes, V., Reis, L., Li, B., Fonte, M. \& De Freitas, M. New approach for analysis of complex multiaxial loading paths. Int. J. Fatigue 62, 21-33 (2014). 